\title{
Disfunción de la coherencia central en niños con trastorno de aprendizaje procedimental
}

\author{
Nerea Crespo-Eguílaz, Juan Narbona, Sara Magallón
}

Introducción. Los niños con trastorno de aprendizaje procedimental (TAP) tienen dificultades para percatarse rápidamente de la coherencia o incoherencia de un material complejo y describirlo verbalmente.

Objetivo. Estudiar la capacidad de escolares con TAP para percatarse de las incoherencias presentes en dos imágenes complejas.

Sujetos y métodos. Se muestran el test de imagen quimérica y la escena visual incoherente a 200 escolares: 20 afectos de TAP, 60 con trastorno por déficit de atención/hiperactividad (TDAH), 60 con TDAH + TAP y 60 escolares típicos como grupo control. Se comparan las respuestas de todos los grupos mediante chi al cuadrado. Se calcula la sensibilidad, eficiencia y especificidad de cada prueba por separado y se realiza análisis discriminante según el rendimiento conjunto en ambas.

Resultados. El rendimiento en los dos grupos con TAP es inferior al de los grupos control y TDAH, tanto en el test de imagen quimérica como en la escena visual incoherente. El 93 y el 92\% de los niños que, respectivamente, rinden bajo en el test de imagen quimérica y en la escena visual incoherente están afectos de TAP (con o sin TDAH comórbido). El 87\% de los sujetos con TDAH + TAP falla en una de las pruebas o en ambas, mientras que sólo lo hace el 15\% de los niños con TDAH.

Conclusiones. Las dificultades específicas para la comprensión rápida, simultánea y coherente de imágenes o escenas complejas, que forman parte del perfil neuropsicológico del TAP, son detectables mediante el test de imagen quimérica y la escena visual incoherente. Estos instrumentos se han revelado altamente sensibles, eficaces y específicos.

Palabras clave. Aprendizaje no verbal. Aprendizaje procedimental. Coherencia central. Escena compleja incoherente. Extinción sensorial. Imagen quimérica. Simultagnosia. Trastorno de la coordinación.

\section{Introducción}

El trastorno de aprendizaje procedimental (TAP) -o trastorno de aprendizaje no verbal- afecta a la automatización de rutinas cognitivas y de destrezas perceptivomotoras que se usan sin especial esfuerzo habitualmente. En los afectos de TAP, las citadas rutinas y destrezas se convierten en procesos trabajosos que demandan coste atencional y de la memoria de trabajo, lo que va en detrimento de la comprensión rápida de situaciones en las que hay que manejar información compleja. Por consiguiente, en el TAP se observa trastorno del desarrollo de la coordinación y de la integración visuoespacial, dificultad en los aprendizajes escolares, lentitud en los procesos, y dificultad en la comprensión y el ajuste de la conducta social. En tres de cada cuatro niños con TAP se asocia también trastorno de atención, y es más frecuente el subtipo sin hiperactividad [1,2]. Además, la confrontación rápida de informaciones concurrentes, bajo la modalidad visual, es una tarea especialmente difícil en los niños con TAP $[1,3]$.
Para comprender una situación o una escena es necesario reconocer los elementos que la configuran, integrarlos en un todo y dentro de un contexto, dotar de significado al conjunto y comprobar su verosimilitud (coherencia o incoherencia). El reconocimiento de una imagen visual necesita la interacción entre los sistemas visuales occipitoparietal (vía del 'dónde') y occipitotemporal (vía del 'qué') [4]; y de la confrontación con los sistemas de memoria semántica en los que intervienen el lóbulo frontal y el sistema hipocampotalamocortical $[5,6]$. La función de coherencia central (perceptiva y cognitiva) permite manejar la información de forma global e integrarla en su contexto; es decir, facilita pasar del reconocimiento de los elementos de un conjunto al significado coherente del todo, y viceversa [7]. Y la realización rápida de esta tarea es fundamental en el aprendizaje y en la conducta social.

Presentar una coherencia central débil significa tener un estilo cognitivo caracterizado por la tendencia al procesamiento de los detalles y por la dificultad para integrar la información en un todo co-
Unidad de Neurología Pediátrica. Departamento de Pediatría. Clínica Universidad de Navarra. Pamplona, Navarra, España.

Correspondencia:

Dra. Nerea Crespo Eguílaz. Unidad de Neuropediatría. Clínica Universidad de Navarra. Avda. Pío XII, s/n. E-31080 Pamplona (Navarra).

E-mail:

necrespo@unav.es

Financiación:

Trabajo subvencionado por la Fundación Fuentes Dutor-ICT (Pamplona).

Aceptado tras revisión externa: 26.07.12.

Cómo citar este artículo: Crespo-Eguílaz N, Narbona J, Magallón S. Disfunción de la coherencia central en niños con trastorno de aprendizaje procedimental. Rev Neurol 2012; 55: 513-9.

(C) 2012 Revista de Neurología 
Tabla I. Estadísticos descriptivos de la muestra. Porcentaje por sexo en cada grupo. Media y desviación estándar (entre paréntesis) del cociente intelectual (CI) en los grupos de la muestra clínica, estimado con las escalas de Wechsler para niños IV (WISC-IV) o revisada (WISC-R).

\begin{tabular}{|c|c|c|c|c|c|c|}
\hline & \multicolumn{2}{|c|}{ Sexo } & \multicolumn{4}{|c|}{ Nivel intelectual } \\
\hline & Niños & Niñas & $\mathrm{Cl}$ total & Escala verbal (Cl) & Escala no verbal (CI) & Discrepancia \\
\hline Control $(n=60)$ & $34(56,7 \%)$ & $26(43,3 \%)$ & $104(10,2)^{a}$ & - & - & - \\
\hline TDAH $(n=60)$ & $48(80,0 \%)$ & $12(20,0 \%)$ & $105(13,6)$ & $105(10,9)$ & $104(13,2)$ & 1 \\
\hline $\operatorname{TAP}(n=20)$ & $17(85,0 \%)$ & $3(15,0 \%)$ & $85(10,5)$ & $94(9,9)$ & $82(11,3)$ & 12 \\
\hline TAP + TDAH $(n=60)$ & $41(68,3 \%)$ & $19(31,7 \%)$ & $86(8,8)$ & $96(10,7)$ & $81(12,5)$ & 15 \\
\hline Total $(n=200)$ & $140(70,0 \%)$ & $60(30,0 \%)$ & 104 & - & - & - \\
\hline
\end{tabular}

TAP: trastorno de aprendizaje procedimental; TDAH: trastorno por déficit de atención/hiperactividad. Escala verbal: Cl verbal de la WISC-R o comprensión verbal de la WISC-IV; escala no verbal: CI manipulativo de la WISC-R o razonamiento perceptivo de la WISC-IV; discrepancia: diferencia entre las escalas verbal y no verbal. a ${ }^{\mathrm{Cl}}$ calculado con el test de vocabulario en imágenes Peabody.

herente. Y, en consecuencia, implica tener problemas para identificar lo relevante de lo superfluo en una situación, describir lo importante de una escena, captar lo esencial de una historia sin detenerse en los detalles nimios, reconocer el sentido de las palabras ambiguas según el contexto, comprender una ironía, un chiste, una broma o el lenguaje figurado, etc. En definitiva, tener una coherencia central deficitaria conlleva dificultades para la comprensión contextual de las situaciones sociales y para la adaptación conductual apropiada en ellas [7-9].

Frith [7] sugiere que la falta de coherencia central en los niños con autismo puede explicar algunos de los déficits no explicados por la teoría de la mente $[7,10]$. En la bibliografía, numerosas investigaciones hacen referencia al déficit de coherencia central en el autismo y en el síndrome de Asperger $[7,8,11,12]$. Pero esta disfunción no es exclusiva del trastorno de la gama autista; puede observarse también en el retraso mental y en el TAP $[1,3,13]$ o trastorno de la coordinación con dificultad de comunicación social [14].

Las pruebas más usadas en investigación para valorar la coherencia central utilizan material verbal $[8,15,16]$. En nuestra experiencia clínica resultan útiles dos tareas de percepción visual que requieren una percatación rápida de las incoherencias que en ellas se representan y la elaboración de la consiguiente explicación verbal. El objetivo de esta investigación es comprobar la sensibilidad, eficiencia y especificidad de estas pruebas como medidas de coherencia central, habilidad que está afectada en los niños con trastorno de aprendizaje procedimental.

\section{Sujetos y métodos}

La muestra de este estudio está constituida por 200 escolares (140 niños y 60 niñas) de edades comprendidas entre 6 y 12 años.

La muestra clínica la forman 140 pacientes de la unidad de neurología pediátrica de nuestro centro. La distribución por grupos experimentales es la siguiente: 20 sujetos diagnosticados de TAP, 60 sujetos que cumplen criterios de trastorno por déficit de atención/hiperactividad (TDAH), y 60 sujetos afectos de TAP y TDAH comórbidos (TAP + TDAH). Las frecuencias por sexo se detallan en la tabla I. Los criterios para diagnóstico del TAP son los señalados en el Manual diagnóstico y estadístico de los trastornos mentales, cuarta edición (DSM-IV) para el trastorno de la coordinación; para el diagnóstico de TDAH se han seguido también los criterios del DSM-IV.

Se ha utilizado un grupo control de la población general formado por 60 sujetos (el $57 \%$ varones) del mismo rango de edad.

Todos los sujetos presentan un desarrollo cognitivo normal (cociente intelectual, CI > 85; rango: 85-140), evaluado a través de las escalas de inteligencia Wechsler para niños IV o revisada [17]. Los participantes del grupo control presentan un CI en el test de vocabulario en imágenes Peabody-III [18] comprendido entre 93 y 140 (CI medio: 104).

Los sujetos del grupo con TDAH muestran un perfil cognitivo homogéneo. Sin embargo, como cabía esperar, se encuentran diferencias significativas entre las habilidades verbales y las no verbales, 
a favor de aquéllas, en los niños afectados de TAP -con y sin TDAH- (Tabla I).

La proporción de sujetos según los subtipos de TDAH descritos en el DSM-IV es la que sigue: el $76,7 \%$ de los pacientes del grupo con TDAH presenta la forma combinada de dicha afectación, frente al $23,3 \%$ que muestra predominio de inatención. Cuando el déficit de atención está asociado al TAP, se observa una distribución inversa: 20 sujetos (33\%) exhiben la forma combinada y 40 (66\%), la forma con predominio de inatención.

Se han utilizado las pruebas test de imagen quimérica y escena visual incoherente en la valoración neuropsicológica de todos los niños de la muestra:

- Test de imagen quimérica. Consiste en la percepción e identificación de un dibujo quimérico diseñado para este trabajo (mitad izquierda: cabeza de un pez; y mitad derecha: cuerpo de un cerdo), que se presenta al sujeto durante dos segundos de exposición. Mediante respuesta verbal, el sujeto expresa si ha identificado los dos, uno o ninguno de los animales que forman la quimera y la incoherencia que existe entre las partes de ésta (Fig. 1).

- Escena visual incoherente. Consiste en la comprensión y descripción verbal libre de la lámina M-VII3a de la batería 'medida de inteligencia', de Terman y Merrill [19], en la que está representada una acción: un hombre aparece cortando la rama de un árbol, pero está sentado encima de la parte de la rama que va a caer cuando termine de cortarla, por lo que caerá él al suelo también. La comprensión de este relato visual con su final inferido implica hacerse cargo de la situación global de forma simultánea, rápida y directa.

Para el tratamiento estadístico de los datos de esta investigación hemos empleado el programa SPSS v. 15.0 y hemos utilizado los siguientes procedimientos estadísticos: en las variables cualitativas o categóricas, calculamos las frecuencias absolutas y realizamos las comparaciones entre los grupos independientes mediante $\chi^{2}$ de Pearson y estadístico exacto de Fisher. Se comprueba la validez (efectividad y eficiencia) de las pruebas utilizadas.

\section{Resultados}

\section{Test de imagen quimérica}

Se registra si, tras lo dos segundos de exposición de la imagen, el sujeto reconoce y nombra los dos animales que constituyen el objeto de la quimera, o si
Figura 1. Test de imagen quimérica 'perdo', pez-cerdo (J. Narbona, diseñado para este estudio).

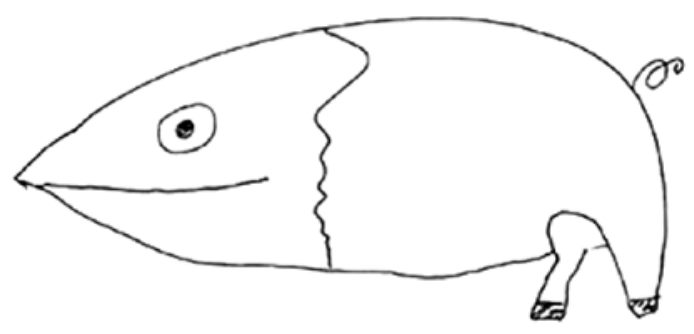

uno o los dos animales no son reconocidos por el sujeto.

Un porcentaje significativamente alto $(85 \%)$ de los componentes del grupo TAP y del grupo TAP + TDAH (68\%) omite el reconocimiento de alguna de las mitades de la figura quimérica. El rendimiento entre los dos grupos citados no es diferente; sin embargo, ambos difieren $(p<0,01)$ del grupo control (un $5 \%$ de omisiones) y del grupo con TDAH (un $8,3 \%$ de omisiones) (Tablas II y III).

Se ha calculado con qué frecuencia son omitidas la mitad izquierda o derecha de la imagen quimérica (correspondientes al hemiespacio derecho e izquierdo ante el sujeto): no existen diferencias en cuanto al hemiespacio ignorado en ninguno de los cuatro grupos de sujetos, ni en las comparaciones intergupos. Esto indica que la dificultad manifiesta en el trastorno de aprendizaje procedimental no es exclusivamente perceptiva, ya que, en ese caso, sería esperable mayor inatención visual al contenido del hemiespacio izquierdo frente al sujeto, lo que no sucede en la presente casuística.

De todos los sujetos de la muestra, el 9,5\% percibe incorrectamente la imagen quimérica, aunque sin negligencia unilateral. Algunos ejemplos de las respuestas erróneas son: 'hámster,' 'pez con patas de perro,' 'mezcla de pez y rinoceronte', 'cara de ballena, cuerpo de jabalí, patas de jabalí, 'pez con patas de elefante', 'hipopótamo, más flaco y con los dientes afilados,' 'una rana, 'como un castor.' Los 19 sujetos que manifiestan este rendimiento se distribuyen en la misma proporción en los diferentes grupos de la muestra y, por tanto, no se aprecian diferencias relevantes según el grupo de pertenencia.

\section{Escena visual incoherente}

Se considera la comprensión de la escena como co- 
Tabla II. Logro obtenido en las pruebas estudiadas: omitir el reconocimiento de una de las dos mitades que componen la imagen quimérica (derecha e izquierda: indica el hemicampo no percibido) y manifestar una comprensión incorrecta de la escena visual.

\begin{tabular}{|c|c|c|c|}
\hline & \multicolumn{2}{|c|}{$\begin{array}{l}\text { No reconocimiento de una } \\
\text { mitad de la imagen quimérica }\end{array}$} & $\begin{array}{l}\text { Comprensión incorrecta } \\
\text { de la escena visual }\end{array}$ \\
\hline \multirow{3}{*}{ Control $(n=60)$} & \multirow{3}{*}{$3(5 \%)$} & Derecha $=1$ & \multirow{3}{*}{$4(6,7 \%)$} \\
\hline & & & \\
\hline & & Izquierda = 2 & \\
\hline \multirow{3}{*}{$\mathrm{TDAH}(n=60)$} & \multirow{3}{*}{$5(8,3 \%)$} & Derecha $=3$ & \multirow{3}{*}{$4(6,7 \%)$} \\
\hline & & & \\
\hline & & Izquierda = 2 & \\
\hline \multirow{3}{*}{$\operatorname{TAP}(n=20)$} & \multirow{3}{*}{$17(85 \%)$} & Derecha $=10$ & \multirow{3}{*}{$15(75 \%)$} \\
\hline & & & \\
\hline & & Izquierda = 7 & \\
\hline \multirow{3}{*}{$\mathrm{TAP}+\mathrm{TDAH}(n=60)$} & \multirow{3}{*}{$41(68,3 \%)$} & Derecha $=25$ & \multirow{3}{*}{$43(71,7 \%)$} \\
\hline & & & \\
\hline & & Izquierda = 16 & \\
\hline
\end{tabular}

TAP: trastorno de aprendizaje procedimental; TDAH: trastorno por déficit de atención/hiperactividad.

rrecta si su descripción incluye tanto la acción presente como su consecuencia inferida: 'un hombre está cortando una rama y cuando termine de cortarla se caerá él al suelo porque está sentado sobre el trozo de rama que se va a desprender.' El 93,3\% de los componentes del grupo control y del grupo con TDAH realiza una descripción correcta, en torno a este contenido, con diferentes palabras, habiendo integrado todos los componentes de la escena simultánea y rápidamente, con una adecuada comprensión de la esencia del relato. En cambio, sólo el $25 \%$ de los sujetos con TAP y el 28,3\% de los afectos de TAP + TDAH logran correctamente esta tarea (Tabla II). No hay diferencias a este respecto entre los dos grupos con TAP, mientras que sí son significativas $(p<0,01)$ al comparar estos dos últimos grupos con los grupos TDAH y control (Tabla III).

Las 58 descripciones incorrectas realizadas por los niños de los grupos TAP y TAP + TDAH pueden ser sistematizadas así:

- El 65,8\% describe la acción, pero no su consecuencia ('un hombre estaba cortando la rama de un árbol para hacer leña'; 'un chico se ha subido a un árbol para cortar la rama'; 'un chico está cortando una rama arriba del todo con una sierra. Y no sabe cómo bajar'). Ante la pregunta realizada por la examinadora '¿y qué va a pasar después?', el $86 \%$ de éstos inventa un final de la historia descontextualizado ('hará fuego'; 'que le pondrán una multa, ¡no está bien cortar las ra-
Figura 2. Análisis discriminante: agrupación de los 80 sujetos (a) con trastorno de aprendizaje procedimental (TAP) de la muestra -TAP y TAP + trastorno por déficit de atención/hiperactividad (TDAH) - y 120 sujetos (b) sin dicho trastorno (control y TDAH), según la interpretación correcta (+) o incorrecta (-) de la imagen quimérica y de la escena visual. El $88,8 \%$ de los sujetos con TAP y el $12,4 \%$ de los sujetos sin TAP fallan una de las pruebas o las dos. El 11,3\% de los niños con TAP y el $87,5 \%$ de los participantes sin TAP manifiestan un rendimiento normal en ambas pruebas. Sensibilidad: 0,88; especificidad: 0,89.
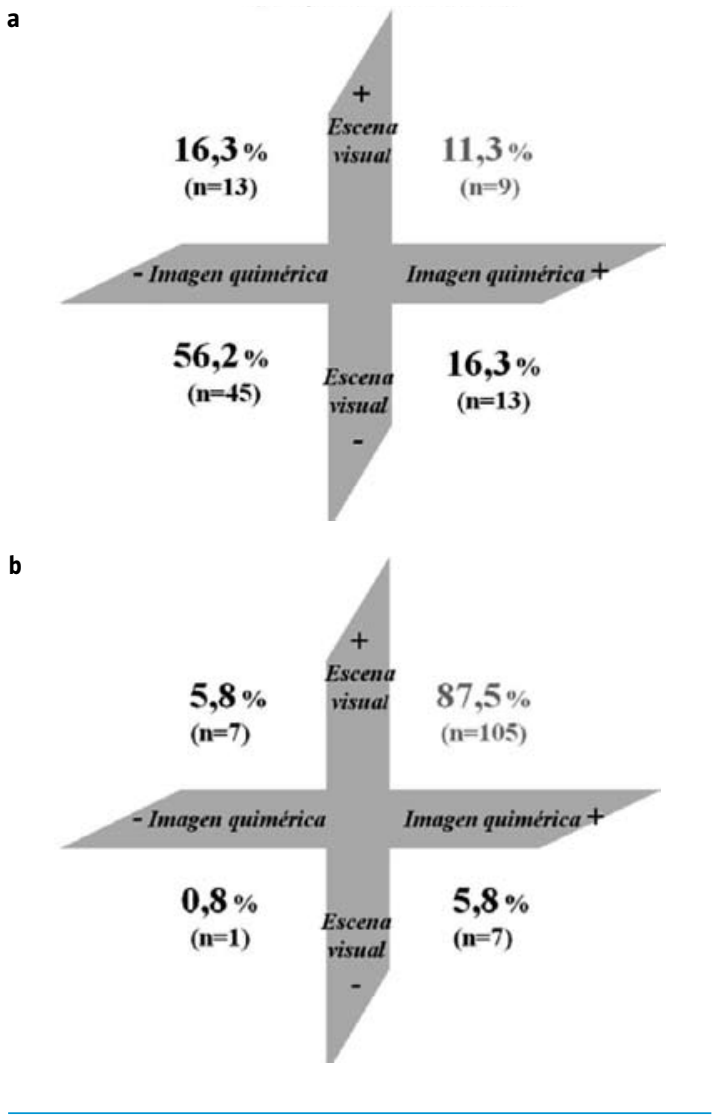

mas de los árboles!'; ‘ ¿cómo va a bajar? tiene que saltar, ¿y si se cae cuando salta?, ¿cómo ha subido?') y sólo un $14 \%$ se da cuenta entonces del desenlace correcto del relato ('AAh! se caerá el hombre, ¿no?'; ‘isi se va a caer!').

- El 22,4\% relata una historia coherente, incluso imaginativa, aunque no relacionada con el relato representado en el dibujo ('un hombre necesitaba madera para hacer fuego porque su mujer tenía una enfermedad; se le congelaban los huesos y las articulaciones. Por eso cortó las ramas de todos los árboles del pueblo, y de todos los pueblos, así la hoguera estaría siempre encendida y su mujer no sufriría'; 'un chico tenía un árbol junto a su 
Tabla III. Diferencias en imagen quimérica y en escena visual entre los grupos control, TDAH, TAP y TAP + TDAH (técnica estadística utilizada: $\chi^{2}$ de Pearson).

\begin{tabular}{|c|c|c|c|c|c|c|}
\hline & \multicolumn{3}{|c|}{ Imagen quimérica } & \multicolumn{3}{|c|}{ Escena visual } \\
\hline & TDAH & TAP & $\mathrm{TAP}+\mathrm{TDAH}$ & TDAH & TAP & TAP + TDAH \\
\hline Control $(n=60)$ & No significativo & $p<0,01^{a}$ & $p<0,01^{a}$ & No significativo & $p<0,01^{a}$ & $p<0,01^{a}$ \\
\hline TDAH $(n=60)$ & - & $p<0,01^{a}$ & $p<0,01^{a}$ & - & $p<0,01^{a}$ & $p<0,01^{a}$ \\
\hline $\operatorname{TAP}(n=20)$ & - & - & No significativo & - & - & No significativo \\
\hline TAP + TDAH $(n=60)$ & - & - & - & - & - & - \\
\hline
\end{tabular}

TAP: trastorno de aprendizaje procedimental; TDAH: trastorno por déficit de atención/hiperactividad. a Diferencias significativas en un intervalo de confianza al $99 \%$.

ventana. Bajó a cortar una de sus ramas, ya que no le dejaba contemplar el paisaje'; 'se había subido al árbol para cortar la leña porque estaba de camping. Y fueron sus papás, su hermanita y sus primos y también sus abuelos. Empezó a llover y se prepararon para irse a casa').

- El 12,1\% realiza una descripción de los elementos del dibujo ('hay un señor, una sierra, un árbol, ramas...'; 'hay un hombre subido en un árbol, el árbol tiene ramas, las ramas no tienen hojas...'; 'un hombre, una sierra, hierba, un árbol roto...').

- Dos pacientes realizan una respuesta inicial que denota literalidad en la comprensión verbal. El primero de ellos, ante la pregunta '¿qué te parece este dibujo?', contesta: 'Bien, es bonito'. El segundo, ante la instrucción 'cuenta lo que sucede', responde: 'uno, dos, tres....'

\section{Estudio de la sensibilidad, eficiencia y especificidad del test de imagen quimérica y de la escena visual incoherente}

Con el fin de comprobar si el rendimiento en estas pruebas aporta una medida eficaz para la diferenciación clínica de los sujetos con TAP, hemos comparado los grupos con TAP y TAP + TDAH con el grupo control en ambas pruebas. Pretendemos confirmar la clasificación correcta de los participantes con arreglo a los siguientes criterios:

- Sujetos con TAP con dificultades para comprender la imagen quimérica y sujetos controles con rendimiento medio en dicha prueba.

- Pacientes diagnosticados de TAP con incomprensión de la escena visual y niños del grupo control con comprensión correcta de la escena. Los resultados se presentan en la tabla IV.
Se puede afirmar que las dos pruebas analizadas en este estudio poseen una sensibilidad alta, mientras que su eficiencia y su especificidad son moderadas. El 93-94\% de los sujetos con negligencia espacial unilateral en la imagen quimérica y el 91-94\% de los niños que no comprenden la escena visual tienen TAP; y sólo un 6-7\% y un 6-8\%, respectivamente, de los que fallan pertenece al grupo control (Tabla IV). La eficiencia nos indica, como ya se ha señalado en los apartados anteriores, que un porcentaje alto del grupo con TAP tiene dificultades en la imagen quimérica (85\%) y en la escena visual $(75 \%)$, como también sucede en el grupo con TAP + TDAH (el $68 \%$ rinde bajo en imagen quimérica y el $72 \%$ en escena visual) (Tabla IV). Por último, la especificidad matiza que un alto porcentaje de los sujetos que rinden correctamente en estas pruebas pertenece al grupo control.

Se ha realizado también el análisis discriminante de las dos pruebas de forma conjunta. Responden incorrectamente, por lo menos, una de las pruebas: el $10 \%$ de los controles, el $15 \%$ de los niños con TDAH, el $87 \%$ de los sujetos con TAP + TDAH y el 95\% de los afectos de TAP. De estos resultados se puede inferir, de nuevo, que los niños con déficit de atención sin trastorno de aprendizaje comórbido rinden bien, por lo general, en las pruebas utilizadas. Para reducir la complejidad de los datos en el análisis discriminante, se consideran dos grandes grupos: el de todos los pacientes con TAP (esto es, los grupos TAP y TAP + TDAH; $n=80$ ) y el de todos los participantes sin dicha patología (es decir, los grupos control y TDAH; $n=120$ ). Como se aprecia en la figura 2 , un alto porcentaje de los niños con TAP $(88,8 \%)$ manifiesta dificultades en una de las tareas o en ambas (sólo el 11,3\% rinde bien en las 
Tabla IV. Sensibilidad, eficiencia y especificidad en el proceso de clasificación de los sujetos (de los grupos señalados) según el rendimiento en imagen quimérica y en escena visual. Ambas pruebas presentan una sensibilidad alta y una eficiencia y una especificidad moderadas como medidas eficaces para la clasificación clínica de los sujetos con trastorno de aprendizaje procedimental.

\begin{tabular}{|c|c|c|c|}
\hline & & $\begin{array}{l}\text { TAP frente } \\
\text { a control }\end{array}$ & $\begin{array}{c}\text { TAP + TDAH } \\
\text { frente a control }\end{array}$ \\
\hline \multirow{3}{*}{$\begin{array}{l}\text { Test de imagen } \\
\text { quimérica }\end{array}$} & Sensibilidad (VP/VP + FN) & 0,94 & 0,93 \\
\hline & Eficiencia (VP/VP + FP) & 0,85 & 0,68 \\
\hline & Especificidad (VN/VN + FP) & 0,86 & 0,77 \\
\hline \multirow{3}{*}{$\begin{array}{l}\text { Escena visual } \\
\text { incoherente }\end{array}$} & Sensibilidad (VP/VP + FN) & 0,94 & 0,91 \\
\hline & Eficiencia (VP/VP + FP) & 0,75 & 0,72 \\
\hline & Especificidad (VN/VN + FP) & 0,79 & 0,77 \\
\hline
\end{tabular}

FN: falsos negativos; FP: falsos positivos; TAP: trastorno de aprendizaje procedimental; TDAH: trastorno por déficit de atención/hiperactividad; VN: verdaderos negativos; VP: verdaderos positivos.

dos). Por otra parte, una elevada proporción de niños sin TAP $(87,5 \%)$ muestra un rendimiento normal tanto en imagen quimérica como en escena visual. Por consiguiente, el análisis discriminante aporta que las dos pruebas aplicadas clasifican correctamente al $88 \%$ del total de los sujetos de la muestra.

\section{Discusión}

La interpretación rápida de una imagen quimérica es dificultosa para el 85\% de escolares con TAP, frente a sólo el $5 \%$ de los sujetos control. En el grupo con TAP + TDAH, el 68,3\% falla en esta tarea, frente al $8,3 \%$ de los afectos de TDAH sin trastorno de aprendizaje comórbido. Se puede deducir que, en la patología aquí referida (TAP con o sin déficit de atención), ocurriría una forma sutil de extinción sensorial del contenido de un hemicampo visual ante la presentación rápida de una imagen quimérica, que no representaría sólo una dificultad atencional, sino, sobre todo, una disfunción ejecutiva referida a la comprobación de verosimilitud del conjunto con dos mitades incoherentes entre sí. Ante este conflicto, el niño con TAP opta por centrarse en interpretar una de las dos mitades (y, por tanto, no presta atención a la otra mitad) para tratar de entender la extraña imagen. Cuando esto sucede, el sujeto conserva la noción de que el dibujo era extraño; lo que no suele suceder en la heminegligencia o en la extinción sensorial clásicas, en las que el paciente no suele ser consciente de su déficit [20-22]. También pue- de interpretarse la dificultad observada como una forma de simultagnosia, descrita como parte del síndrome de Balint en pacientes con lesión o disfunción bilateral de las estructuras parietooccipitales y también en síndromes frontales [23,24].

$\mathrm{Al}$ analizar la descripción de la escena visual incoherente realizada por los integrantes de la muestra, se pone de manifiesto que más del $70 \%$ de los escolares afectos de TAP tienen dificultades en la comprensión de información visual simultánea. Sin embargo, el 7\% de los niños con TDAH y el 7\% de los controles experimentan bajo rendimiento en esta tarea. Por consiguiente, esta dificultad para captar la incongruencia representada en el dibujo, según los datos de este estudio, caracteriza al TAP. El sujeto con TAP tiende a centrarse sobre algún detalle del conjunto y a pasar de un detalle a otro en su descripción, sin realizar una apreciación del conjunto, lo que motiva su dificultad para descubrir la incoherencia de la escena. Se han documentado en la patología aquí referida (TAP con y sin déficit de atención) alteraciones en el reconocimiento de los detalles, en el procesamiento simultáneo, en la organización visuoespacial y en la integración de las partes en un todo [1,2,25].

Una limitación del presente estudio es la utilización de sólo dos tareas conflictivas visuales; probablemente la simultagnosia y la disfunción de coherencia central que aquí se perfilan como formando parte del TAP puedan, en lo sucesivo, ser estudiadas de forma más refinada aplicando series más numerosas (y graduadas en cuanto a dificultad) de imágenes conflictivas, así como de material verbal. Pero en la investigación presente, el análisis discriminante de los resultados en el test de imagen quimérica y en la escena visual incoherente pone de relieve que, de forma conjunta, el rendimiento en estas dos tareas clasifica correctamente al $88 \%$ de los sujetos que cumplen criterios de TAP frente a los que no cumplen tales criterios. Además, los grupos con TAP + TDAH y TDAH difieren en el rendimiento en las dos pruebas estudiadas. Por consiguiente, si un niño con déficit de atención tiene dificultades en estas dos pruebas, o en una de ellas, estaría indicado indagar la posible presencia de criterios de TAP [2].

Los hallazgos de esta investigación apuntan que ambas pruebas son medidas eficaces para evaluar la función de coherencia central en el TAP $[3,7]$.

\footnotetext{
Bibliografía

1. Gillberg C. Deficits in attention, motor control, and perception: a brief review. Arch Dis Child 2003; 88: 904-10.

2. Crespo-Eguílaz N, Narbona, J. Trastorno de aprendizaje
} 
procedimental: características neuropsicológicas. Rev Neurol 2009; 49: 409-16.

3. Crespo-Eguílaz N, Narbona J. Dificultades en la percatación rápida de incongruencias en el trastorno de aprendizaje procedimental: posible disfunción de la coherencia central. Rev Neurol 2011; 52 (Supl 1): S39-41.

4. Ungerleider LG, Mishkin M. Two cortical visual systems. In Ingle D, Goodale M, Mansfield R, eds. Analysis of visual behavior. Cambridge: MIT Press; 1982. p. 549-86.

5. Atkinson J, Braddick O. Visual and visuocognitive development in children born very prematurely. In Von Hofsten C, Rosander K, eds. Progress in brain research. Vol. 164. Amsterdam: Elsevier; 2007. p. 123-49.

6. Fazzi E, Bova S, Giovenzana A, Signorini S, Uggetti C, Bianchi P. Cognitive visual dysfunctions in preterm children with periventricular leukomalacia. Dev Med Child Neurol 2009; 51: 974-81.

7. Frith U. Autism: explaining the enigma. Oxford: Basil Blackwell; 1989.

8. Noens I, Berckelaer-Onnes I. The central coherence account of autism revisited: evidence from the ComFor study. Res Autism Spectr Disord 2008; 2: 209-22.

9. López B, Leekam, S. Teoría de la coherencia central: una revisión de los supuestos teóricos. Infancia Aprendizaje 2007; 30: 439-57.

10. Happé F, Frith U. The weak coherence account: detail-focused cognitive style in autism spectrum disorders. J Autism Dev Disord 2006; 1: 5-25.

11. Van Lang N, Bouma A, Sytema S, Kraijer D, Minderaa R. A comparison of central coherence skills between adolescents with an intellectual disability with and without comorbid autism spectrum disorder. Res Dev Disabil 2006; 27: 217-26.

12. Le Sourn-Bissaoui S, Caillies S, Gierski F, Motte J. Ambiguity detection in adolescents with Asperger syndrome: is central coherence or theory of mind impaired? Res Autism Spectr Disord 2011; 5: 648-56.

13. Gillberg C. Developmental and neuropsychiatric disorders of childhood. In Aicardi J, Bax M, Gillberg C, eds. Diseases of the nervous system in childhood. 3 ed. London: Mac Keith Press; 2009. p. 889-901.

14. American Psychiatric Association. DSM-5 Development. URL: http://www.dsm5.org/pages/default.aspx. [01.05.2012].

15. Booth R, Happé F. 'Hunting with a knife and... fork': examining central coherence in autism, attention deficit/ hyperactivity disorder, and typical development with a linguistic task. J Exp Child Psychol 2010; 107: 377-93.

16. Jolliffe T, Baron-Cohen S. A test of central coherence theory: linguistic processing in high-functioning adults with autism or Asperger syndrome: is local coherence impaired? Cognition 1999; 71: 149-85.

17. Wechsler D. Escala de inteligencia Wechsler para niños IV (WISC-IV). Madrid: TEA; 2005.

18. Dunn LM, Dunn L, Arribas D. PPVT-III Peabody Test de vocabulario en imágenes. Adaptación y baremo españoles. Madrid: TEA; 2006.

19. Terman L, Merrill MA. Medida de inteligencia. Madrid: Espasa Calpe; 1976.

20. Posner MI, Gilbert CD. Attention and primary visual cortex. Proc Natl Acad Sci U S A 1999; 96: 2585-7.

21. Heilman K, Watson R, Valenstein E. Neglect and related disorders. In Heilman K, Valenstein E, eds. Clinical neuropsychology. 3 ed. New York: Oxford University Press; 1993.

22. Gabari I. Desarrollo neuropsicológico en sujetos con lesión cerebral unihemisférica congénita. Rev Psicol Gen Apl 1995 48: 301-14

23. Farah MJ, Feinberg TE. Apperceptive agnosia and simultagnosia. In Feinberg TE, Farah MJ, eds. Behavioral neurology and neuropsychology. New York: McGraw-Hill; 1997. p. 239-41.

24. Rafal RD. Balint syndrome. In Feinberg TE, Farah MJ, eds. Behavioral neurology and neuropsychology. New York: McGraw-Hill; 1997. p. 337-56.

25. Rourke B, Ahmad S, Collins D, Hayman-Abello B, HaymanAbello S, Warriner E. Child clinical/pediatric neuropsychology: some recent advances. Annu Rev Psychol 2002; 53: 309-39.

\section{Dysfunction of central coherence in schoolchildren with procedural learning disorder}

Introduction. Children with coordination disorder -non verbal learning disorder, procedural learning disorder (PLD)- have difficulties in understanding complex simultaneous visual information.

Aim. Validation of two different visual tasks to measure central coherence function of children with PLD.

Subjects and methods. A chimeric image and a complex visual story are showed to 200 schoolchildren: 20 of them have PLD, 60 have PLD plus attention deficit/hyperactivity disorder (PLD + ADHD), 60 have non comorbid ADHD, and 60 subjects are typical control children. A chi square test and a discriminant analysis are used to study the performances of the different groups in verbal description of both images.

Results. Performance is lower in children with PLD and PLD + ADHD than in those with non-comorbid ADHD or controls. Moreover $93 \%$ and $92 \%$ of children with poor performance in, respectively, chimeric and complex images, have PLD or PLD + ADHD. Eighty seven per cent of subjects with PLD + ADHD fail in some of the tasks and, by contrast only $15 \%$ of children with ADHD do.

Conclusions. Children with PLD have disability in quick understanding of simultaneous complex information and central coherence. The two tasks used in this research are useful to detect these difficulties, with high sensibility and specificity.

Key words. Central coherence function. Coordination disorder. Non verbal learning disability. Procedural disorder. Simultagnosia. Strange images. Visual extinction. 